Nouvelles perspectives en sciences sociales

\title{
Perspectives de recherche et d'action pour la valorisation scientifique : sur quelques expériences hypermédias en archéologie
}

\section{Julien Mahoudeau}

Volume 3, numéro 1, septembre 2007

URI : https://id.erudit.org/iderudit/602464ar

DOI : https://doi.org/10.7202/602464ar

Aller au sommaire du numéro

\section{Éditeur(s)}

Prise de parole

ISSN

1712-8307 (imprimé)

1918-7475 (numérique)

Découvrir la revue

Citer cet article

Mahoudeau, J. (2007). Perspectives de recherche et d'action pour la valorisation scientifique : sur quelques expériences hypermédias en archéologie. Nouvelles perspectives en sciences sociales, 3(1), 9-46. https://doi.org/10.7202/602464ar

\section{Résumé de l'article}

La communication des savoirs scientifiques est un élément essentiel de la science, participant de son ampleur et de ses justifications politiques et sociales. Centrée sur le champ de l'archéologie, la réflexion se focalise ici sur la valorisation hypermedia entendue comme processus de mise en valeur par les techniques digitales des savoirs scientifiques à destination de publics nonspécialistes. Quatre expériences de conception et réalisation d'hypermédias de valorisation du patrimoine archéologique servent de socles pour explorer différentes perspectives : complexité endogène des hypermedias, professionnalisation du médiateur hypermedia, transversalité des dispositifs, complexité exogène. Nous proposons que cette conjonction de perspectives aide à mieux comprendre comment sont définies de nouvelles conditions de recherche et d'action pour la valorisation scientifique. Nous voulons aussi montrer concrètement comment les techniques digitales participent à l'avancée de la science et à la démocratisation des connaissances par le biais d'outils de médiation. 


\title{
Perspectives de recherche et d'action pour la valorisation scientifique : sur quelques expériences hypermédias en archéologie
}

\author{
JULIEN MAHOUdEAU \\ Université toulousaine d'archéologie et d'histoire \\ UMR 5608 CNRS
}

« La communication scientifique est devenue un outil stratégique de première importance, car elle permet d'informer l'opinion publique et les décideurs politiques sur les développements concernant des sujets sensibles ». Cette déclaration du Conseil de l'Europe (rapport de la Commission de la culture, de la science et de l'éducation du 18 décembre 2001)' met en exergue l'importance effective du savoir scientifique et de sa mise en communication. Il est vrai qu'elle concerne plus spécifiquement les sciences dites dures et les situations où le savoir scientifique sert de fondement à une action / décision politique. Mais elle illustre cependant bien la conscience générale de l'importance sociale et politique de la communication scientifique, quelles que soient les formes qu'elle prend. Globalement, ce phénomène particulier de communication s'inscrit dans un projet politique et social qui vise à

Citée dans : Claude Le Bouf et Nicolas Pelissier, "Communiquer dans la complexité », dans Claude Le Bœu uf et Nicolas Pelissier (dir.), Conımuniquerl'information scientifique - F'tbique du joumalisme et stratégies des organisations, Paris, L'Harmattan - Série communication et technologie, 2003, p. 13-18. 
favoriser un rapprochement de la science et des citoyens. Ce projet part du principe que l'information scientifique n'est pas destinée à un cercle restreint de spécialistes, mais concerne au contraire un très large public. Le fondement sociologique de ce principe est double : d'une part, l'objectif même de la science, qui est la connaissance pour mieux comprendre le monde et mieux vivre en son sein, ne peut se concevoir comme clos sur lui-même, hors du monde, et le savoir scientifique se conçoit démocratiquement dans un processus de transmission et d'appropriation collective des connaissances produites ; d'autre part, nombre de secteurs de la vie courante sont concrètement en interaction avec les avancées de la science. Pour ces deux raisons au moins, la communication des savoirs scientifiques est un élément essentiel de la science, participant de son ampleur et de ses justifications politiques et sociales. En matière d'archéologie comme en d'autres sciences, la communication des connaissances produites dans le cadre de pratiques professionnelles de recherche est une part intrinsèque d'une activité qui se mène en quasi-totalité dans la sphère publique, une part déontologique, pourrait-on dire. Au sein des problématiques de la communication scientifique, on s'intéresse ici à la valorisation hypermédia entendue comme processus de mise en valeur par les techniques digitales des savoirs scientifiques à destination de publics non spécialistes. À partir d'un recadrage préalable de ce dont on traite sous les vocables de médiation et de valorisation comme processus spécifique de communication, quatre expériences de conception et réalisation d'hypermédias de valorisation du patrimoine archéologique servent de support aux réflexions proposées.

La première expérience concerne la création d'un CD-Rom autour du site archéologique de Montsérié dans le département français des Hautes-Pyrénées. Ce CD-Rom correspond à une expérience de développement d'un hypermédia pédagogique en relation avec un site archéologique de portée locale, connu par un corpus d'autels votifs ${ }^{2}$ romains, mais porteur d'une histoire beaucoup plus longue et complexe que la seule occupation romaine attestée. Le site est dominé par un

Offrandes faites aux dieux dans les temples par les fidèles et qui prennent la forme canonique d'un petit édifice de pierre sur lequel le fidèle inscrit son action d'offrande. 
oppidum ${ }^{3}$ et présente des traces d'occupation datant du Bronze Ancien (XXII ${ }^{c}$ XVII ${ }^{c}$ siècles av. J.-C.) et allant jusqu'au XVIII siècle de notre ère. Il présente la particularité de n'avoir jamais été fouillé par des équipes scientifiques. Un partenariat s'est monté avec une enseignante de latin d'un collège de Tarbes qui faisait travailler ses élèves sur l'écriture romaine au travers des autels votifs conservés au musée Massey de Tarbes et provenant du site de Montsérié. Le CD-Rom, qui met en valeur le patrimoine archéologique autour de quelques éléments géographiques et historiques, accompagne les élèves latinistes dans leur découverte à la fois de l'épigraphie latine et d'un site archéologique fortement porteur d'histoire locale. La deuxième expérience concerne la réalisation d'un hypermédia servant de support à une conférence scientifique. Dans le cadre de la mission franco-tunisienne de fouille à Zama ${ }^{4}$, qui travaille sur l'adduction d'eau dans la ville antique, j'ai été chargé de travailler auprès des équipes archéologiques sur le terrain de fouille à la mise en place de processus de valorisation hypermédia du patrimoine archéologique issu des fouilles. Pour accompagner une conférence destinée à présenter les résultats des recherches, une première réalisation a été développée sous la forme d'un support visuel à un discours scientifique oral, sorte de CD-Rom que manipule l'orateur pour illustrer son propos. La troisième expérience concerne la réalisation d'un site $\mathrm{Web}^{5}$ autour d'un site archéologique d'Albanie, le site de Sovjan, en partenariat avec l'École Française d'Athènes. Situé dans le bassin de Korçë et fouillé depuis 1993 par une équipe francoalbanaise, Sovjan est un site d'habitats lacustres pré-historiques et protohistoriques pour lequel nous avons pu être intégré à l'équipe sur le terrain pour travailler à la valorisation du travail scientifique archéologique de la mission. La dernière expérience concerne la

\footnotetext{
Espace fortifié pré-romain.

Situé en Tunisie, Zama est le lieu de la dernière bataille de la Deuxième Guerre Punique opposant, en 202 av. J.-C., le général carthaginois Hannibal au général romain Scipion l'Africain et consacrant la victoire quasi définitive de Rome sur Carthage.

http ://www.sovjan-archeologie.net, site consulté en juillet 2007.
} 
réalisation d'un site $\mathrm{Web}^{6}$ destiné à mettre en valeur le patrimoine archéologique du castrum Bigorra - Castelbieilh de Saint-Lézer (HautesPyrénées). Pour promouvoir son patrimoine et mettre en valeur les résultats de dix années d'investigations archéologiques et architecturales, la Communauté de communes de Vic-Montaner, sur le territoire de laquelle est situé le site, a passé commande de la réalisation d'un dispositif hypermédia?.

Les quatre expériences relatées ici ne sont que quelques-unes, significatives, parmi nombre d'autres que nous avons pu réaliser, issues d'une pratique professionnelle de médiation hypermédia du patrimoine, et qui servent aussi de bases aux réflexions proposées ici'. Ces données empiriques servent de socles pour mener des réflexions autour des processus de circulation des connaissances par les techniques digitales. En s'attachant à quelques points particuliers déroulés au fil de la présentation des expériences, elles sont l'occasion d'emmener le lecteur vers la conjonction de différentes perspectives : complexité endogène des hypermédias (perspective cognitiviste et problématique de conception), professionnalisation du médiateur hypermédia, transversalité des dispositifs, complexité exogène (perspective sociologique)". C'est cette conjonction de perspectives qui nous semble permettre de mieux comprendre comment sont définies de nouvelles conditions de recherche et d'action pour la valorisation scientifique. Nous voulons aussi montrer concrètement comment les techniques digitales participent à l'avancée de la science et à la démocratisation des connaissances par le biais d'outils de médiation.

\footnotetext{
http://www.vic-montaner.com/patrimoine/archeologie/index.htm, site consulté en juillet 2007

On s'étendra moins sur la description de ces deux derniers dispositifs, dans la mesure où ils sont consultables en ligne.

* Rappelons également que le champ des réalisations est immense, un très grand nombre de dispositifs de médiation étant en ligne sur Internet. L'observation active, l'analyse du champ des productions hypermédias culturelles, dont cet article ne rend pas compte, alimentent également notre propos. Des analyses de productions auxquclles nous n'avons pas participé auraient tout aussi bien permis de nourrir les quelques réflexions proposées.

Ces différentes réflexions sont rattachées une par une aux expériences préscntées, mais pourraient toutes s'appliquer à chacune d'entre elles.
} 


\section{Sur la communication, la médiation et la valorisation des savoirs scientifiques}

Au sein des processus de communication, on travaille ici sur la médiation, terme qui recouvre d'une manière générale l'idée d'un intermédiaire se trouvant entre un savoir et un récepteur de ce savoir. C'est un terme générique que nous inscrivons dans un processus de transmission d'un savoir, mais sans que cette définition contienne intrinsèquement la description de l'objectif précis de la transmission. Nous nous focalisons alors sur la vulgarisation ou, de préférence, la valorisation scientifiques.

Le terme latin vulgare est un terme très général, qui contient de prime abord une connotation négative, corrélativement au terme vulgus renvoyant à la catégorie du peuple, à laquelle renvoie également un autre terme utilisé en la matière : populariser. La vulgarisation met ainsi en jeu le clivage culturel et social entre le scientifique et le citoyen. «Il y a une liaison entre les deux substantifs dérivés de l'adjectif : la vulgarité met à distance la culture d'un autre social et la vulgarisation exprime l'effort délibéré pour faire partager à l'autre sa culture propre, réputée universelle ${ }^{10} »$. Mais en dépit de l'utilisation du mot pour faire référence aux travaux importants en la matière, ne faut-il pas préférer le mot valorisation, qui donne au concept sa portée culturelle et épistémique, au mot vulgarisation, qui les dégrade ? En effet, si les significations sont strictement équivalentes ${ }^{11}$, la valorisation a pour elle l'atout de la connotation positive. Elle renvoie davantage à la valeur du processus de transmission des connaissances scientifiques qu'à ce qu'il a de vulgaire. En outre, elle indique bien que ce processus peut être source de valeur pour la science elle-même. On utilisera donc ici les termes de valorisation et ses dérivés préférentiellement à celui de vulgarisation.

La valorisation scientifique est une pratique de discours autour des savoirs scientifiques. Selon une première conception, elle concerne un

\footnotetext{
Yves Jeanneret, Écrire la science - Forme et sens de la 1utgarisation, Paris, PUF, 1994, p. 14.

Sans ignorer que l'expression «valorisation de la recherche scientifique 》 possède également une connotation économique, et désigne alors une activité d'inscription des résultats de la recherche scientifique dans des circuits socioéconomiques.
} 
processus de diffusion dont le but est de partager les savoirs, partage qui peut être vu comme son objet central : rendre accessibles des connaissances spécialisées à des non-spécialistes au moyen d'une médiation, contribuer au développement de l'intelligibilité du monde pour le plus grand nombre. Elle relève alors d'une composante politique qui vise à recréer un lien communautaire entre le monde des sciences et la société, à restaurer une appartenance commune entre citoyens et scientifiques. Vue comme une transposition didactique, elle peut s'envisager dans le cadre spécifique des processus et contextes d'enseignement, d'apprentissage, de relation pédagogique. La transmission y est clairement déterminée par un objectif pédagogique d'acquisition de savoirs, compétences, références, repères, etc. Sur ces deux versants politique et didactique, le discours de valorisation scientifique cherche à favoriser une reconstruction des connaissances dans les milieux récepteurs. La notion de reconstruction est ici essentielle : elle indique que la valorisation met en jeu une activité d'interprétation des énoncés scientifiques, dont elle n'est jamais seulement une reproduction en des termes plus simples ${ }^{12}$. Mais nous reconnaitrons alors en jeu une question épistémologique de première importance : cette dimension d'inter-prétation n'est pas le propre de la valorisation, elle est déjà constitutive de l'activité scientifique elle-même. Pour peu qu'on veuille bien s'attacher à une épistémologie non positiviste $^{13}$, on peut considérer que les résultats de la science ne sont pas des vérités objectives, mais sont eux-mêmes des constructions de savoirs, qui disent autant de chose sur le chemin emprunté pour parvenir au résultat que sur le résultat lui-même. Ils sont des interprétations du monde, certes bâties à partir d'une démarche rigoureuse qui fait le propre de l'activité scientifique et qu'il n'est pas question de nier, mais qui n'en restent pas moins des interprétations du

\footnotetext{
Yves Jeanneret illustre ainsi cette idée de l'interprétation, c'est-à-dire de la production d'un nouveau discours, par la problématique de la répartition, en vulgarisation, du savoir et de l'ignorance : « chaque stratégie de discours est un choix plus ou moins conscient sur ce que l'on fait savoir et ce qu'on laisse ignorer", Yves Jeanneret, op. cit., p. 80.

1.3 Voir par exemple Gaston Bachelard, Le nounel esprit scientifique, Paris, PUF, 1934, ou Jean-Touis Le Moigne, Le constructivisme. tomes 1 à 3, Paris, L'Harmattan, coll. "Ingénium », 2001, 2002, 2003.
} 
réel. L'histoire des sciences tout entière témoigne de ce que les énoncés scientifiques sont profondément dépendants des contextes dans lesquels se déroule la recherche, des paradigmes dominants à un moment donné de l'histoire, des positions socio-culturelles de ceux qui les formulent ${ }^{14}$.

Sur ces questions se développe alors la problématique du « fait de langage ${ }^{15}$ » comme composante de l'activité de valorisation. La science peut dire quelque chose du monde d'une manière qui lui est propre, tout comme la valorisation peut dire quelque chose de la science (et donc du monde) d'une manière qui lui est propre. Il ne s'agit pas de nier le fait que science et valorisation construisent des discours de nature différente, mais de reconnaître l'existence des faits de langage comme constitutive de ces deux domaines, tout comme le sont les processus d'interprétation. En tant que processus de traduction, la valorisation peut alors être vue comme ayant pour but de rendre la science intelligible en traduisant le langage scientifique, incompréhensible pour le néophyte, en langage courant. «La vulgarisation comme traduction, c'est la conscience que le cognitif est avant tout du linguistique, que notre connaissance est structurée comme un langage ${ }^{16} \%$. Ce qui renvoie aux problématiques propres des terminologies scientifiques et à la manière dont elles s'élaborent (qui n'est pas plus objective que celle des vulgarisateurs). Yves Jeanneret montre l'importance, en valorisation scientifique, des faits de langage, du fait littéraire et de l'hétérogénéité linguistique. Remarquons cependant que l'ésotérisme des langages apparait souvent davantage comme un rituel de défense corporative que comme nécessité épistémique a priori.

Une autre question importante se pose à la valorisation : la science transportée hors du champ scientifique reste-t-elle de la science? Ce qu'Yves Jeanneret nomme "la philosophie du soupçon ${ }^{17}$ " est ainsi portée par l'idée que les savoirs ne se transmettent qu'en s'altérant ${ }^{18}$. Le

4 Voir par exemple Gaston Bachelard, L a formation de l'esprit scientifique, Paris, V rin, 1938, ou Thomas Kuhn, La structure des révoiutions scientifiques, Paris, Flammarion, 1972.

Yves Jeanneret, op. cit.

Ibid., p. 32.

Ibid., p. 51-66.

1* Idée illustrée, par exemple, dans l'ouvrage de Philippe Roqueplo, Le partage des savoirs. Science, culture, vulgarisation, Paris, Seuil, 1974. 
vulgarisateur n'est-il qu'un médiateur entre le public et la communauté scientifique, médiateur qui, par nature, dénature la science? Pour certains auteurs, la valorisation appartient au contraire pleinement au champ scientifique. Ainsi Daniel Jacobi ${ }^{19}$ suggère-t-il qu'il y a un continuum qui lie les démarches, les intentions et finalement les textes des scientifiques et des vulgarisateurs. Cette idée conduit à considérer que la valorisation participe de la science en ce qu'elle permet l'émergence d'une certaine manière de penser la science. Reconnaittre ce rôle social spécifique de la valorisation est en même temps lui reconnaître de fortes implications épistémologiques : la valorisation scientifique peut formuler des points de vue sur la science que les pratiques scientifiques spécialisées n'élaborent que très rarement elles-mêmes; elle peut donner au chercheur, de l'extérieur, une meilleure connaissance de sa discipline et un regard critique sur son activité scientifique. Sur un autre versant, deux idées complémentaires permettent d'étoffer la complexité ici entrevue des rapports entre science et valorisation. D'une part, la valorisation peut être vue comme participant au rayonnement de la science ; mais l'attitude des scientifiques à l'égard de la valorisation balance malgré tout entre un rejet, parfois virulent, une acceptation fataliste ou bien un franc enthousiasme. D'autre part, notre société, où l'information est considérée comme une ressource stratégique, encourage la valorisation. Cette tendance se ressent de plus en plus dans la définition stratégique des programmes de recherche, où il est demandé aux scientifiques, en amont des recherches, de porter une attention toute particulière aux opérations de diffusion de leurs futurs résultats. La valorisation ne saurait faire l'impasse sur les processus de réception du sens des messages valorisés et ne saurait se passer de réfléchir sur les intentions qui vont motiver ceux qui consultent les ouvrages (ou utilisent les dispositifs) de valorisation. Quel est le sens de la volonté de savoir ? La compréhension de ce qui motive l'acquisition de connaissances, mais aussi la prise en compte des modalités de réception des savoirs valorisés (dans quels cadres ? quels lieux ? quelles conditions?) apparaissent fondamentales.

19. Daniel Jacobi, Diffusion et vulgarisation : itinéraire du texte scientifique, Annales littéraires de l'Université de Besançon, Les Belles Lettres, 1986. 
Ainsi, il faut légitimement reconnaitre un statut particulier au discours de valorisation, aux limites du champ scientifique, mais participant peutêtre plus qu'il n'y paraît de prime abord à l'activité scientifique. Ces éléments de cadrage étant posés, examinons maintenant les expériences en archéologie proposées comme données empiriques permettant d'articuler quelques réflexions sur les nouvelles perspectives de recherche et d'action pour la valorisation scientifique hypermédia.

\section{L'hypermédia comme dispositif pédagogique : le site archéologique de Montsérié}

\subsection{Genèse et sens du projet}

Situé dans les Hautes-Pyrénées, le site archéologique de Montsérié est un site de piémont qui connaît une occupation humaine depuis l'époque au moins protohistorique. Redécouvert au $\mathrm{XIX}^{\mathrm{e}}$ siècle au travers des vestiges et du mobilier d'un sanctuaire indigène de l'époque romaine (vestiges parmi lesquels se trouvait un masque célèbre dit à tort pendant longtemps "Masque de Tarbes » et conservé au musée Massey de Tarbes), le site est aussi dominé par les traces d'une fortification de type oppidum, pourvu en son secteur central de vestiges mégalithiques et couvert sur son flanc sud de traces d'occupations agro-pastorales presque certainement médiévales. Présentant une configuration géographique malcommode (large couvert forestier, grande dispersion des vestiges, escarpement important), entouré de prestigieux sites archéologiques $^{20}$, le site de Montsérié n'a jamais été fouillé ni approché dans son ensemble.

À l'origine, l'attention a été portée sur ce site au travers d'un projet d'action pédagogique hypermédia. Comme nous l'avons dit, c'est la rencontre avec une enseignante de Latin du collège Massey, qui faisait travailler ses élèves sur l'épigraphie latine à partir du corpus d'autels votifs romains provenant de Montsérié, qui a conduit à imaginer un tel projet : bâtir un hypermédia didactique en liaison avec un site archéologique complexe non fouillé et un matériel archéologique spécifique et

2" St-Bertrand-de-Comminges, site de l'antique L,ugdunum Convenarum, ou encore Grotte préhistorique de Gargas. 
non représentatif de l'ensemble du site (autels votifs romains). Un CDRom, aujourd'hui publié par le Centre départemental de documentation pédagogique (CDDP) de Tarbes, a donc été réalisé, prolongé par un dispositif pédagogique complet comprenant plusieurs visites au musée, un déplacement sur le site archéologique et plusieurs séances de travail en classe, sur ordinateur, autour du CD-Rom. Celui-ci se fixait comme objectif de transmettre des éléments de connaissances d'histoire locale et d'archéologie en resituant les objets étudiés par les élèves en cours de latin et au musée dans leur environnement archéologique d'origine. Les élèves concernés ont été deux classes de $4^{c}$ et de $3^{c}$ du collège Massey de Tarbes. L'équipe de conception s'est composée de l'enseignante de Latin (définition globale du projet, accompagnement des élèves pour les visites au musée, sur le site et les séances d'utilisation du CD-Rom en classe) ; l'attaché de conservation pour l'archéologie au Musée Massey (inventaire des objets de Montsérié conservés au musée, accompagnement des visites au musée et sur le site) ; un infographiste (conception graphique) ; l'auteur de cet article (conception globale, arborescence, recherche et rassemblement de la documentation, prospection sur le site, rédaction des contenus, couverture photographique, réalisation technique du CD-Rom, accompagnement des élèves sur le site et lors des séances d'utilisation du CD-Rom); à quoi il faut ajouter le soutien actif de la Commune et du Maire de Montsérié (aide sur le terrain, prospection, mise à disposition du matériel archéologique pour étude).

\subsection{Déroulement de l'expérience pédagogique}

L'expérience pédagogique elle-même s'est décomposée en plusieurs temps successifs. Tout au long de l'année, dans le cadre du cours de latin, les élèves ont approché la religion romaine et l'épigraphie latine, au travers des dédicaces des autels votifs. Plusieurs séances au musée Massey ont permis de travailler directement devant les objets. Lors d'un cours spécifique, le CD-Rom est présenté aux élèves par une projection sur grand écran, juste avant d'aller visiter le site. L'accent est mis sur la partie présentant les vestiges visibles. L'objectif est de replacer la connaissance du site dans un environnement complexe et de préparer les élèves à la visite en facilitant leur appréhension des vestiges. À ce stade, les élèves ne manipulent pas encore eux-mêmes le CD-Rom, qui 
leur est simplement présentée ${ }^{11}$. Les élèves effectuent ensuite avec l'équipe pédagogique une visite du site et abordent la réalité du terrain d'un site difficile et, ce faisant est mise en avant l'idéc selon laquelle la connaissance du passé lointain ne peut venir que de fouilles archéologiques scientifiques. Par la suite, le CD-Rom est installé sur les postes de la salle informatique du collège. L'outil hypermédia permet alors aux élèves de compléter leur visite et d'approfondir leurs connaissances. Ainsi, une partie importante du CD-Rom permet-elle, sous forme de fiches accompagnées d'éléments interactifs, de revenir sur toutes les questions soulevées par la présence à l'époque romaine d'un sanctuaire dans lequel est honoré un dieu indigène. Il reste alors à mesurer, pour les auteurs du projet, l'impact des hypermédias sur l'assimilation de la culture archéologique par un jeune public, et pour l'enseignante, à évaluer les connaissances acquises par les élèves en ce qui concerne la civilisation romaine. Cette évaluation a été réalisée au collège, après la visite du site et l'utilisation du CD-Rom, par des interrogations écrites.

\subsection{Objectifs pédagogiques}

L'axe majeur de la médiation consistait à replacer des objets observés au musée dans un environnement archéologique complexe, en utilisant les potentialités de l'outil hypermédia pour travailler à plusieurs objectifs conjoints.

D'une part, à partir d'une connaissance particulière (les autels votifs de la période romaine), élargir le champ de connaissance historique (périodes précédentes et postérieures à l'occupation romaine), c'est-àdire travailler à faire comprendre que ce qu'on peut étudier ou connaittre à partir d'un corpus ou d'un thème n'est toujours qu'un secteur restreint de la réalité historique. En l'occurrence, s'agissant d'éléments d'histoire

\footnotetext{
En effet, dans ce cas précis, si l'hypermédia doit être utile en amont de la visitc physique du site, il ne nous a pas semblé pertinent de mettre d'emblée le CD-Rom entre les mains des élèves. Ce choix se justifie par la relative complexité du CD-Rom lui-mème, dont nous avons pensé qu'une présentation préalable aiderait à la manipulation après la visite du site archéologique. Mais c'est aidés d'une préparaţion par un outil hypermédia qu'ils abordent la découverte des vestiges sur le terrain, notamment de l'espace fortifié protohistorique.
} 
locale (et donc peut-être émotionnellement proche des élèves), le site archéologique révèle des occupations indigènes bien antérieures à la période romaine, et le thème de connaissance n'est plus seulement «un site des Pyrénées à la période romaine ", mais peut devenir aussi "l'occupation indigène pré-romaine ", " les traces du processus de romanisation dans le piémont pyrénéen », ou encore «l'activité agropastorale médiévale ». Le site de Montsérié se prête particulièrement bien à ce genre d'exercice d'élargissement cognitif « horizontal », c'est-à-dire entendu dans le champ chronologique.

D'autre part, il fallait renforcer la connaissance d'un secteur particulier de l'histoire, qui reste le point d'accroche du projet : Montsérié à l'époque romaine. En effet, le cours de Latin, donc l'épigraphie latine, reste dans un tel projet le point d'entrée nécessaire. À travers les autels votifs, ce sont en fait des éléments de la culture romaine qui sont à l'étude. L'hypermédia est particulièrement bien adapté pour présenter une connaissance construite en séquences non linéaires et se déclinant en thèmes interconnectés. À partir de l'objet " autel votif romain ", de nombreuses questions peuvent être légitimement évoquées : qu'est-ce qu'un sanctuaire ? à quoi sert-il ? à quoi servent ces autels? que nous disent-ils de la religion et de la culture de ceux qui les ont produits? etc. Autant d'approches constituant des " unités d'information » qu'il faut articuler dans un hypermédia permettant un élargissement cognitif « vertical », c'est-à-dire entendu comme approfondissement d'un thème d'étude donné.

\subsection{Principes de navigation et de conception hypermédia}

Le CD-Rom s'organise en quatre parties, qui ont chacune un objectif précis : a) une approche générale du site, qui permet de le situer géographiquement et de comprendre l'historique de sa découverte; b) une partie présentant les vestiges visibles sur le site. Elle permet à la fois de préparer les élèves à la visite, et d'approfondir celle-ci par la suite. Le paradoxe de cette partie a été de présenter la complexité d'un site archéologique difficile à appréhender et dont la connaissance scientifique nous fait défaut ; c) une partie sur Montsérié à l'époque romaine. Organisée en fiches qui tentent de répondre à un certain nombre de questions, elle replonge les élèves dans tout ce qui a été étudié en cours d'année à partir des objets du musée et en classe de latin ; d) une partie 
"Ressources pédagogiques", qui contient de nombreux documents pédagogiques utilisables par l'enseignant et les élèves (notamment l'ensemble des fiches en format imprimable ainsi que la collection numérique).

Un « Menu principal » situé én partie inférieure gauche permet à l'élève de naviguer au sein des quatre parties. Par un passage de la souris sur l'outil « Menu principal », il fait apparaittre la liste des quatre parties, qui sont alors mises en surbrillance, et affiche les pages concernées par un clic sur chacun des quatre titres de partie. Chacune est identifiée par un code couleur qui se retrouve dans l'ensemble du CD-Rom. $\grave{A}$ l'intérieur de chacune d'entre elles, le sous-menu est alors présenté en partie inférieure droite. Dans l'ensemble des pages, la partie gauche est occupée par le texte tandis que la partie droite présente les éléments d'illustration graphique.

À l'intérieur de chaque page, les éléments importants sont mis en avant par des jeux interactifs : par le passage de la souris sur les éléments textuels en bleu ou sur l'illustration, l'élève fait apparaître des éléments graphiques explicatifs. Décrivons ici l'exemple des pages concernant les autels votifs.

Nous nous sommes d'abord attachés à faire comprendre comment se constitue et se décrit un autel votif. Par un passage de la souris sur le dessin de l'autel ou sur les éléments textuels le décrivant, l'élève peut voir distinctement chacune des parties constitutives de l'autel votif : la base, le corps, le couronnement, les moulures, le coussin. Dans la page suivante, nous nous sommes attachés à faire comprendre comment se constituent les dédicaces inscrites sur les autels votifs. À partir d'un exemple d'autel, le passage de la souris sur l'image met en relief chacun des éléments de la dédicace : le nom du dieu à qui l'autel est dédié (ici le dieu Mars : Marti Deo), le nom du dédicant (ici Colius), puis la formule traditionnelle indiquant que l'offrande a été faite telle qu'elle devait l'être (ici la formule stéréotypée VSLM, V (otum) S(olvit) L(ibens) $\mathrm{M}$ (erito), signifiant « s'est acquitté de ce vœu de bon gré, comme de juste »). Dans les pages présentant les vestiges visibles, plusieurs principes graphiques ont été utilisés. L'idée est toujours la même, celle de la mise en avant des éléments par un passage de la souris sur les illustrations graphiques ou sur le texte associé. Pour certaines images, le passage de la souris fait apparaître un dessin qui vient en surimpression : c'est par exemple le cas pour l'endroit supposé du sanctuaire de l'époque 
romaine. Celui-ci est en effet constitué d'une terrasse formée par un grand mur de soutènement. Ce choix de dessins en surimpression se justifie par la difficulté de lecture des images ${ }^{22}$. Pour d'autres éléments, les photographies permettent de distinguer correctement les vestiges. Le jeu d'interactivité permet alors de mettre en avant les différents éléments repérables. Ceux-ci sont cernés d'un trait rouge, les autres parties étant opacifiées. Une seule page utilise une animation réalisée à l'aide du logiciel Flash de Macromédia : elle concerne les levées de terre visibles sur le site traduisant la présence de fortifications. L'animation fait apparaitre en surimpression un dessin permettant d'imaginer ce que pouvait être la fortification de l'oppidum. Enfin, un peu partout dans les pages du CD-Rom, des jeux interactifs permettent d'afficher, au passage de la souris, des éléments d'informations complémentaires. C'est par exemple le cas pour donner des définitions ou approfondir une idée.

L'ensemble du CD-Rom a été développé à l'aide du logiciel de création HTML Dreamweaver 4.0 de Macromédia. Les éléments graphiques ont été travaillés à l'aide du logiciel Photoshop d'Adobe. Les jeux d'interactivité sont créés à l'aide de plusieurs fonctions du logiciel Dreamweaver, qu'ils s'appliquent à du texte ou à des images ${ }^{23}$.

\section{5. Évaluation}

Dans le cadre de ce projet, l'évaluation concernait l'appréciation des connaissances réellement acquises par les élèves au travers de l'utilisation du CD-Rom. Nous avons pu constater un intérêt manifeste

Le site n'étant pas fouillé, le couvert forestier et végétal est total sur le site et nous avons pensé que ces dessins pouvaient aider à mieux lire les photographies des vestiges.

23 Pour appliquer un jeu interactif à une image, la première étape consiste à créer sous Photoshop les différentes images qui seront activées par le passage de la souris. Lnsuite, sous le logiciel Dreamweaver, il faut procéder à la définition des zones actives. Enfin, en utilisant l'outil «comportement», on définit, d'une part, la fonction à appliquer, par exemple «invertir une image» (swap image), et d'autre part, les actions de l'utilisateur qui déclencheront cette fonction, par exemple le passage de la souris (mouse over et mouse out). Pour appliquer un jeu interactif à un élément textuel, nous utilisons le mème outil "comportement", avec cette fois la fonction "affichermasquer les calques " (sbow/bide layers), et toujours la dćfinition des évènements auxquels s'applique la fonction. 
des élèves non seulement pour le thème étudié, pour l'histoire locale et l'archéologie mais aussi pour l'utilisation de l'outil informatique. Cet intérêt s'est traduit à la fois par une grande attention en classe lors des séances d'utilisation et par une forte attention lors de la visite du site et lors des visites au musée. D’une manière générale, on peut distinguer différentes «catégories » d'élèves : a) sur une classe d'une vingtaine d'élèves, seuls deux ont semblé éprouver de réelles difficultés de concentration et donc d'appréhension des contenus ; b) une grande majorité d'entre eux semble avoir compris l'essentiel des contenus, bien que ces élèves n'accèdent cependant pas toujours aux détails les plus complexes qui sont proposés ; c) seul un petit nombre d'élève est capable de repérer les éléments les plus complexes contenus dans le CD-Rom (par exemple les questions traitant d'onomastique).

D'une manière générale, les éléments d'interactivité permettent à l'évidence aux élèves de se sentir impliqués dans la construction des connaissances : c'est par leur action au moyen de la souris que les éléments didactiques apparaissent à l'écran. L'hypermédia permet en outre à chaque élève de progresser à son propre rythme dans les contenus. Des différences significatives en termes de temps d'appréhension et de facilité d'utilisation du CD-Rom sont apparues entre les élèves possédant un ordinateur chez eux et ceux n'en possédant pas. D'autre part, l'utilisation même d'un hypermédia peut être l'occasion pour l'utilisateur, ici les élèves latinistes, de porter quelques regards sur l'outil de connaissance qu'ils manipulent. S'il est effectivement difficile de provoquer consciemment une telle démarche par le seul jeu de l'hypermédia (particulièrement chez de jeunes élèves plus enclins à être attentifs au côté ludique), l'accompagnement d'un médiateur peut aider les élèves à s'interroger sur la manière dont les informations sont présentées, organisées, mises en mouvement.

En définitive, il nous est apparu que l'expérience pédagogique hypermédia se révélait largement positive. L'utilisation du CD-Rom, alliée à des visites au musée et à une visite du site archéologique, a permis à une grande majorité d'élèves de replacer les autels votifs (objets initialement étudiés en cours de Latin) dans leur environnement d'origine; de comprendre que les vestiges romains n'étaient la trace que d'une partie seulement de l'histoire, et donc qu'il y avait avant l'arrivée des Romains toute une civilisation préromaine dite «indigène »; et de comprendre que l'intérêt des vestiges archéologiques est en l'occurrence 
de nous en apprendre autant sur les coutumes romaines que sur le processus de romanisation des populations du piémont pyrénéen.

\subsection{Vers la reprise scientifique du site archéologique de Montsérié}

Cette expérience permet d'observer un passage de la médiation pédagogique hypermédia à la recherche scientifique archéologique, illustrant par une première facette atypique la participation des hypermédias à l'avancée de la science. Le travail effectué pour la réalisation du CD-Rom nous a en effet incité à proposer un état des lieux de la connaissance scientifique actuelle du site et à les présenter dans un rapport à un laboratoire d'archéologie (l'Unité toulousaine d'archéologie et d'histoire). L'objectif en était de fournir un document synthétique qui permette à une équipe scientifique de démarrer un réel travail de terrain. Une telle opération est aujourd'hui sur le point d'être programmée.

2.7. Complexité endogène des hypermédias : perspective cognitiviste et problématique de conception

Nous proposons ici quelques réflexions relevant d'une perspective cognitiviste : l'ensemble des processus cognitifs mis en jeu par l'opérationnalité manipulatoire d'un hypermédia apparait comme décisif. Car c'est en « jouant » avec les différents outils interactifs proposés que les utilisateurs s'impliquent dans la construction du savoir.

Le champ de l'interactivité se révèle très étendu et malaisé à cerner, la notion s'utilisant dans de nombreux domaines et ne pouvant se réduire à une seule et même définition. Elle est étudiée selon un ensemble d'approches très diverses, auxquelles le lecteur intéressé pourra se reporter: sciences de l'information et de la communication ${ }^{24}$,

2+ Voir, par exemple : Geneviève Vidal, «L'interactivité et les sciences de l'information et de la communication ", Actes du XVII' congrès des sociologues de langue française - Tours, juillet 2004, Groupe de travail Sociologie de la communication, 2004, p. 366-375, ou Françoise Séguy, Lesproduits interactifs et multimédias - Méthodologies - Conception - Écritures, Grenoble, Presscs universitaires de Grenoble, coll. "La Communication en Plus », 1999. 
analyses des discours sur les usages des TIC $^{25}$, analyses des stratégies des acteurs économiques ${ }^{26}$, recherche en informatique sur les interfaces adaptatives, analyses de la charge cognitive, de l'ergonomie et du design des produits interactifs, recherche sur les usages sociocognitifs ${ }^{27}$, travaux sur les médiations techniques ${ }^{28}$, sciences du langage ${ }^{29}$, analyses sémiologiques $^{30}$, sociopolitique des usages ${ }^{31}$, toutes ces démarches apportant des éclairages complémentaires.

Pour le concepteur hypermédia, l'interactivité est un processus de formalisation ou de structuration des contenus à l'écran, mise en forme adaptée aux objectifs de l'hypermédia et à l'utilisateur à qui il s'adresse. Dans les réalisations présentées, l'interactivité est mise au service d'une intention de médiation de contenus archéologiques et culturels. Pour l'utilisateur, elle se conçoit comme un outil d'accès et de manipulation d'informations. De ce point de vue, l'aspect essentiel de l'interactivité

25 Par excmple, sur les croyances relatives à Internet, voir Philippe Breton, Le culte de l'Internet, Paris, La Découverte, 2000, ou dans le cadre de la net-économie : Manuel Castells, La société en réseaux, Paris, Fayard, 1998 et Manuel Castells, La galaxie internet, Paris, Fayard, 2001.

20 Par exemple : Jean-Guy Lacroix et al., "Industries culturelles et informatisation sociale - Nouvelles perspectives de recherche ", Sciences de la société, $\mathrm{n}^{\circ} 40$, Toulouse, PUM, 1997, p. 3-9.

27 Par exemple: Philippe Mallein et al, « Multimédia et significations d'usage : les critères sociologiques de qualité d'usage du multimédia, le cas d'Internet ", dans Michel Dubois (dir.), Etude sociocognitive des usages du multimédia, ERIHST-CERAT / CNRS / CNET, janvier 1998.

2* Par exemple : Madeleine Akrich, «Les formes de la médiation technique », Réseaux, $n^{\circ}$ 60, 1993, p. 87-98.

29 Par exemple : Jean-Thicrry Julia et Emmanuelle Jambert, "Énonciation et interactivité : du réactif au créatif », Communication et langages, $\mathrm{n}^{\circ} 137,2003$, p. 30-44.

3" Par exemple: Yves Jeanneret, Joëlle Le Marec et Emmanuel Souchicr, Lire, écrire, récrire. Objets, signes et pratiques des médias informatisés, Paris, BPI / Centre Pompidou, coll. "Études et recherche », 2003.

3 Par exemple : Pierre Chambat, «Usage des TIC : évolution des problématiques », Technologies de l'information et société, vol. 6, $\mathrm{n}^{\circ} 3,1994$, p. 249-270, ou Josiane Jouët, "Retour critique sur la sociologie des usages", Réseaux, n 100, 2000, p. 487-521, ou encore Serge Proulx et Michel Sénécal, "L'interactivité technique, simulacre d'interaction sociale et de démocratie?", Technologies de l'information et société, vol. 7 , $\mathrm{n}^{\circ} 2,1995$, p. $239-255$. 
demeure celui-ci : elle met en ceuvre des pratiques manipulatoires mobilisant chez l'utilisateur un fonctionnement cognitif spécifique dans lequel la perception des signes et symboles présents à l'écran et les intentions d'action sur ces signes et symboles tiennent une place primordiale. Elle existe au travers d'expériences sensibles, c'est-à-dire d'expérimentations concrètes des dispositifs faisant appel aux sens. Elle se matérialise dans une alternance entre la perception et l'action, alternance qui met en œuvre chez l'utilisateur une certaine capacité d'invention ${ }^{32}$. Cependant, loin de simplifier la tâche humaine, bien souvent les hypermédias la complexifient et augmentent en conséquence la charge cognitive, précisément dans la mesure où ils mettent en œuvre des opérations cognitives d'un ordre nouveau liées à la mise en écran des informations. Les utilisateurs se trouvent plongés dans un environnement informatique entrainant de profondes mutations qui imposent de nouvelles manières de raisonner et d'appréhender les contenus. L'usage des techniques digitales interactives favorise donc un mode d'apprentissage par le faire, où l'action de l'utilisateur est un élément essentiel du processus d'apprentissage. C'est en faisant, en observant à l'écran les résultats d'une action, en manipulant les données, les images, les sons que l'apprentissage est facilité. En outre, les techniques digitales induisent un rapport à l'imaginaire de l'écran qui génère des formes d'accès à la connaissance, de comportement et des attentes particulières et différentes de celles rencontrées pour d'autres médias. Elles offrent ainsi une médiation vers d'autres modes d'apprentissage ('émotion, le jeu). L'utilisation de l'écran a des conséquences sur la manière dont l'information acquise va être enregistrée, emportée et gardée par l'utilisateur. L'appropriation d'un hypermédia (fonctionnalité et contenu) est facilitée par l'intensité de l'investissement personnel et par l'objectif visé par l'utilisateur. D'où l'importance des intentions

Serge Proulx, "Usages des technologies d'information et de communication : reconsidérer le champ d'étude? " dans Émergence et continuité dans les recherches en information et communication - XII Congrès international des sciences de linformation et de la communication, janvier 2001, UNESCO (Paris), SFCIC, 2001, p. 64-65, http://grm.uqam.ca/textes/proulx_SFSIC2001.pdf,p.64-65, site consulté en mars 2007. 
préalables de l'utilisateur ${ }^{33}$ ou du sens qu'il donne aux produits ${ }^{34}$. Le projet d'usage de l'utilisateur conditionne ses attentes et donc son usage. De la nature du projet et de sa charge symbolique dépendra le rapport de l'usager à l'objet technique et son mode d'appropriation.

Encore faut-il voir que ces processus s'exercent sur des objets digitaux créés et conçus par des concepteurs, en d'autres termes que la complexité endogène des hypermédias tient aussi aux questions de conception ${ }^{35}$. Cette conception révèle par exemple le problème crucial de la représentation de l'information. Par principe, le concepteur d'hypermédias de médiation du patrimoine opère des choix pour traiter la façon dont les informations sont représentées, c'est-à-dire mises en forme et offertes à l'utilisateur. Les vecteurs véhiculant l'information, textes, sons, images (fixes ou animées, photographiques ou modèles graphiques) sont les supports de la représentation, celle-ci demeurant de l'ordre de la traduction, de l'interprétation, de la reformulation. Profondément ancrée dans les processus cognitifs de construction du sens, la représentation hypermédia des informations entraîne des modifications majeures des processus cognitifs par lesquels se construisent l'univers des signes, la circulation de l'information, l'élaboration des connaissances, l'organisation des savoirs. Elle conditionne le statut sémiotique des signes qui s'offrent autant à l'interprétation qu'à la manipulation. Acte fondamental d'une écriture hypermédia consciente de son statut, le choix des vecteurs de représentation régit la formalisation du discours de médiation. Ainsi, la définition de l'organisation (aussi appelée l'architecture) des documents hypermédias est un pôle majeur de l'écriture et de la conception. Elle consiste à décider de la structure fondatrice, permettant de distinguer, classer et hiérarchiser les contenus. L'organisation est intimement liée à la définition des parcours proposés, partie de l'écriture hypermédia qui

3.3 On verra, par exemple, la notion de «projet personnel d'usage» dans: Jean Davallon, Hana Gottesdiener et Joëlle Le Marec, «Approche de la construction des usages de cédéroms culturels liés aux musées ", Université Jean Monnet, Centre d'étude et de recherche sur les expositions et les musées, Ministère de la culture, Direction des musées de France / Réunion des musées nationaux, Paris, 1997.

34 On verra ici la notion de «signification d'usage » dans: Philippe Mallein et al., op. cit.

35 Mahoudeau, Julien, Médiation des savoirs et complexité. Le cas des bypermédias archéologiques et culturels, Paris, L'Harmattan, coll. « Ingénium », 2007, p. 105-160. 
décide des enchaînements possibles et de leurs modalités, dimension traditionnellement identifiée par la métaphore de la navigation. L'hypermédia se fonde sur l'idée que l'accumulation et la juxtaposition ne sont jamais suffisantes : c'est l'articulation des unités d'informations et des médias présents qui détermine l'architecture et la navigation. L'écriture, au sens premier du terme, c'est-à-dire de la construction d'un discours de valorisation, est un point crucial de l'écriture hypermédia. Les hypermédias de médiation du patrimoine n'échappent pas aux principes dégagés par les travaux d'Yves Jeanneret ${ }^{36}$ selon lesquels la valorisation scientifique est toujours une affaire d'énonciation, d'écriture au sens littéraire du terme. L'hypermédia ne saurait gommer la complexité des faits de langue liés à l'élaboration et à la transmission des savoirs, en dépit d'une complexité accrue du système d'énonciation luimême. La construction d'un discours garde une place centrale et la notion identifiée par Yves Jeanneret de « contrat de communication" entre le médiateur et le public de la valorisation scientifique reste tout à fait pertinente dans la sphère hypermédia. Il ne saurait y avoir de valorisation hypermédia des savoirs scientifiques archéologiques et culturels sans écriture, sans discours, sans processus d'énonciation, sans faits de langages. L'opérationnalité d'ordre manipulatoire des hypermédias ne dispense en rien les concepteurs d'assumer, en amont de la réalisation technique usant d'interactivité, la définition d'un projet de médiation. Projet dans lequel l'organisation discursive est fondamentale.

2.8. Processus de valorisation et de co-construction des savoirs : la conjonction des projets de conception et d'usage

Ici, nous voulons relever un élément essentiel : c'est la conjonction d'un projet de conception et d'un projet d'usage qui donne son plein sens à un hypermédia de valorisation du patrimoine. En fait, c'est dans cette conjonction que se réalise le processus de valorisation, de diffusion des savoirs. Ainsi, nous proposons d'appréhender les situations de valorisation scientifique comme des systèmes co-construits par les actions des interlocuteurs de la médiation et fondés autour d'un objectif de transmission d'un contenu.

36 Yves Jeanneret, op.cit. 


\section{L'hypermédia comme outil de conférence scientifique : le site archéologique de Zama en Tunisie}

\subsection{Mission franco-tunisienne : présentation et contexte}

L'équipe de recherche franco-tunisienne de Zama travaille depuis 2002 sur le thème de l'alimentation en eau de la ville antique. Les objectifs de la mission de recherche sont multiples. Le premier volet correspond à l'activité " classique » de toute recherche archéologique de terrain : fouilles, étude des vestiges, études architecturales, études du mobilier, plans, relevés, rapports de fouilles, archives images, conclusions historiques. La coopération franco-tunisienne prévoit aussi un volet portant sur la formation, permettant d'ancrer les rapports des équipes tunisiennes et françaises dans la durée et dans une transmission réciproque des savoirs. Enfin, un troisième et dernier volet de la coopération porte sur la mise en valeur du site archéologique de Zama et la préparation à son ouverture aux visites touristiques. La médiation hypermédia se trouve au cœur de ce dernier aspect, qui est directement à l'origine de notre présence au sein de l'équipe française de fouille à Zama. La mise en valeur touristique du patrimoine est en effet l'une des préoccupations importantes des autorités tunisiennes et c'est dans ce contexte que les hypermédias sont envisagés comme produits de valorisation touristico-culturelle. Le projet scientifique se comprend donc aisément.

Derrière l'objectif évident de connaissance historique et archéologique, ce qui nous intéresse est de comprendre et de faire comprendre comment les habitants de Zama, placés dès cette époque dans un cadre naturel difficile, ont su, avec l'aide de leurs techniciens expérimentés de l'hydraulique, maitriser ce milieu et en exploiter toutes les ressources. Archéologie et histoire urbaine, archéologie et histoire de l'environnement, archéologie et histoire des techniques, archéologie et mise en valeur du Patrimoine, telle est en somme la quadruple dimension de cet ambitieux projet ${ }^{3-}$.

37 UTAH, « Recherches sur l'alimentation en eau à Zama dans l'Antiquité. Bilan et perspectives après la campagne de septembre 2003 ", Rapport de la mission tuniso-franfaise de foutlle à Zama, Unité toulousaine d'archéologie et d'histoire, UMR 5608 CNRS, Toulouse, 2003, p. 1. 


\subsection{Zama antique : aperçu géographique et historique}

Le site antique de Zama est situé dans le gouvernorat de Siliana, au centre de la Tunisie, à environ $170 \mathrm{~km}$ au sud-ouest de Tunis. Se tenant immédiatement derrière les premières collines formant les contreforts du Djebel Massouge, le village contemporain de Jama était situé à l'exact emplacement d'un grand temple antique, mis au jour depuis, et a été déplacé à proximité au début des années 1960. La ville est située dans un environnement de collines calcaires avec de nombreuses cultures dans les dépressions et dans la plaine de Siliana, qui est presque certainement le lieu de la bataille de Zama. Le massif calcaire est riche en sources, dont un certain nombre sont captées et utilisées depuis l'Antiquité. La ville de Zama s'inscrit dans une histoire qui remonte à l'époque punique. Celle-ci désigne la période que couvre la civilisation carthaginoise et qui s'étend du début du $1^{\mathrm{er}}$ millénaire av. J.-C. (Carthage est fondée par des colons phéniciens vers 800 av. J.-C.) jusqu'à la victoire définitive de Rome sur Carthage ${ }^{38}$ en 146 av. J.-C. Le site de Zama tient un rôle historique tout à fait important, puisque c'est là qu'eut lieu en 202 av. J.-C. la dernière bataille de la Deuxième Guerre Punique marquant la victoire du général romain Scipion l'Africain sur le général carthaginois Hannibal. Zama fut ensuite ville royale à l'époque numide. À l'époque romaine, elle est devenue ville libre puis, à partir d'Hadrien, colonie romaine. Elle reste une cité importante jusqu'à l'époque byzantine. Le site de Zama a été définitivement identifié par la découverte en 2001 d'une stèle inscrite et dédiée aux "Zamaregienses », c'est-à-dire aux « citoyens de Zama Regia » (« Zama la Royale»), découverte qui a confirmé que le site de Jama était bien celui de la Zama Regia antique.

38 En effet, Rome et Carthage se sont construites presque l'une face à l'autre pendant près de 400 ans, et l'histoire des rapports entre les deux civilisations au cours de la deuxième moitié du premier millénaire avant notre ère n'est qu'une succession de tensions, de conflits et de guerres, nommés pour les plus importants les « Guerres Puniques $\%$. 
3.3. Problématiques scientifiques de la mission : le thème de l'hydraulique dans l'Antiquité

L'alimentation en eau de la ville antique de Zama s'est donc imposée comme thème de recherche. Pour un certain nombre de raisons, il est apparu que Zama représentait pour le type d'investigation envisagée " un objet de recherche privilégié, et presque un cas d'école ${ }^{39}$ »: une importance historique intrinsèque, des sources littéraires antiques concernant Zama qui mettent l'accent, de façon récurrente, sur le problème de l'eau, la permanence du site dans la très longue durée. Le programme scientifique associe prospections, études et sondages dans l'environnement rural de la ville antique. Les problématiques de la mission sont multiples : rechercher activement tous les indices intrinsèques concernant la chronologie relative et absolue des aménagements rencontrés ; comprendre l'organisation des débouchés urbains des aqueducs ; étudier de manière approfondie certains vestiges précis ${ }^{40}$; articuler les autres éléments présents sur le territoire de la ville et à ses abords dont certains ont commencé à apparaître : bassins de décantation, structures de redistribution... et qui vont compliquer et affiner des problématiques forcément envisagées, au départ, de manière un peu schématique. Par ailleurs, il faut relever la volonté des membres de la mission de travailler à un entrelacement de tous les aspects scientifiques avec la valorisation du patrimoine archéologique mis au jour et des résultats des études scientifiques qui en découlent. L'hypermédia est envisagé pour suivre l'évolution des recherches, dont il serait intéressant qu'il les reflète dans leur ensemble, et prend pleinement sa place au sein du travail archéologique scientifique.

Pour cette étude qui s'annonçait très complexe, nous avons rassemblé des compétences diverses, et le document que nous présentons aujourd'hui, malgré les progrès effectués, a un caractère tout à fait provisoire : il reflète l'état actuel de nos connaissances. Celui-ci est enrichi à la fois sur le plan scientifique et sur celui de la valorisation du patrimoine par le recours à

\footnotetext{
UTAH, op. cit., p. 2.
}

+" La galerie de captage d'Ain Ben Ali, le barrage-voûte d'Ain D jebour, le pont de l'oued Krafes et les grandes citernes publiques. 
l'outil informatique et aux réalisations qu'il permet en matière de documents multimédias destinés à divers publics ${ }^{41}$.

\subsection{Valorisation hypermédia à Zama}

La présence d'un médiateur hypermédia au sein de l'équipe de fouille de la mission franco-tunisienne à Zama est donc directement liée à une demande des partenaires tunisiens, qui souhaitent que les équipes étrangères participant à des fouilles en Tunisie consacrent une partie de leurs efforts à la formation des équipes, la conservation et la valorisation des vestiges. Les responsables de la mission ont ainsi estimé que les hypermédias pouvaient être en l'espèce un vecteur important de valorisation du site archéologique. L'intégration du médiateur hypermédia permet de travailler en amont, au plus près des opérations de terrain, à la conception future de produits de valorisation hypermédia. La qualité et la richesse du site archéologique, qui prendront leur sens au cours des années de recherches, complétées par un aménagement du site pour les visites touristiques et par la réalisation d'outils hypermédias de mise en valeur, pourront être à terme de forts atouts de promotion touristique. Car, au-delà de la connaissance scientifique, c'est bien en effet l'un des objectifs majeurs des partenaires tunisiens pour le site de Zama. Cependant, dès les premières campagnes de fouille, disposant sur place d'un ordinateur portable équipé des logiciels adéquats, il a été possible de commencer à stocker un certain nombre de données et à réfléchir à une articulation des informations archéologiques scientifiques au sein d'un hypermédia. Lors de la campagne de fouille d'avril 2003, il a été suggéré à l'ensemble de l'équipe française de donner une conférence à Siliana proposant une présentation de l'état actuel de nos recherches. Celle-ci a été l'occasion de réaliser un premier dispositif hypermédia qui se concevait alors comme support visuel d'une conférence scientifique. Celui-ci ne se conçoit donc pas, ou pas encore, comme un hypermédia "autonome ", livrable au public à qui il s'adresse, et ne contient, en ce qui concerne la présentation des vestiges, aucun texte susceptible de commencer à contextualiser les éléments visuels

+1 UTAH, op. cit. 
présentés. Par ailleurs, précisons que cet hypermédia a été réalisé dans un très court laps de temps et sans les compétences d'un infographiste.

D'un point de vue technique, cette présentation hypermédia est structurée en $\mathrm{HTML}^{42}$, et non au format PowerPoint de Microsoft traditionnellement utilisé pour les communications scientifiques projetées sur grand écran. Outre un degré d'interactivité très largement supérieur au format PowerPoint, l'HTML est naturellement destiné au Web et ne nécessite pour l'utilisateur qu'un navigateur Internet, toujours présent sur les systèmes d'exploitation. Les principes généraux hypermédias de cette réalisation sont les mêmes que ceux utilisés pour le CD-Rom de Montsérié : des jeux interactifs permettent, au passage de la souris, de mettre en avant les éléments essentiels. L'arborescence est relativement simple et suit la présentation du travail effectué autour des différents secteurs et vestiges. Un menu permettant d'accéder à chaque partie est disponible en permanence en partie haute de l'écran.

Ainsi, concernant les grandes citernes, pour lesquelles l'équipe a procédé à des études architecturales du bâti et pas encore à des fouilles, il s'agissait d'illustrer brièvement le fonctionnement de ces structures hydrauliques de stockage de l'eau. Ainsi, les trois termes "recevoir, stocker, distribuer vers la ville » permettent de faire apparaitre sur les photographies des calques illustrant chacune de ces fonctions. La présentation se poursuit par des croquis architecturaux proposant de premières hypothèses sur l'état initial de ces monuments ${ }^{4.3}$. Dans le secteur dénommé Oued Gnadez, des vestiges de deux des aqueducs sont clairement visibles. Le premier jeu interactif permet de bien montrer les deux aqueducs collés l'un à l'autre, tandis que le deuxième jeu de calques permet de bien distinguer les parois, la voûte et la présence d'un enduit hydraulique portant d'importantes concrétions. À la source d'Aïn Slimane, un aménagement moderne capte l'eau. Des rigoles antiques ont été repérées et le jeu interactif est l'occasion

+2 I.es logiciels utilisés ont été le logiciel Photoshop d'Adobe pour le traitement graphique et le logiciel Dreamweaver de Macromédia pour la création HTMI.

+3 "Grâce aux documents de travail multimédia, il est possible de proposer des restitutions tridimensionnelles de cet ensemble monumental. Ces restitutions devront être validées par des sondages à venir mais peuvent également se présenter sous forme de différentes hypothèses analogiquement référées à des exemples locaux ou plus lointains au sein de l'Empire romain » (UTAH, op. cit., p. 26). 
d'illustrer la permanence de l'utilisation de cette source. Dans un autre secteur, l'un des ouvrages d'art importants est un pont aqueduc traversant l'oued Krafes, sur le parcours de l'aqueduc provenant d'Aïn Djebour. Quelques beaux vestiges de piles subsistent encore, et l'ouvrage a fait l'objet d'études architecturales approfondies. Le jeu interactif très simple sert ici à permettre de localiser le pont aqueduc dans son environnement géographique. Enfin, situé à environ une vingtaine de kilomètres de la ville de Zama, l'un des secteurs sur lesquels travaille la mission a permis la découverte des vestiges d'un ouvrage d'art très important : le barrage voûte d'Aïn Djebour, quasi unique dans le monde romain quant à son état de conservation. Celui-ci, qui a fait l'objet de nettoyages, de prospections, de sondages et d'études préliminaires, sera encore l'objet de toutes les attentions de l'équipe de recherche. En particulier, des fouilles sont à prévoir qui seules permettront d'apporter des éléments de connaissance relatifs au fonctionnement réel de cette structure, à ses différents états et périodes de construction et consolidation.

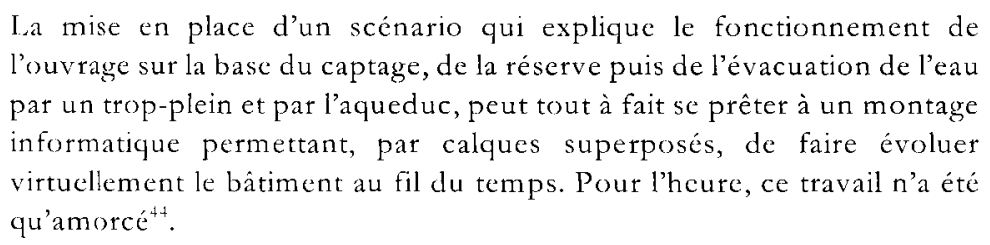

Le jeu de calques interactifs est utilisé ici à différents niveaux. Dans un premier temps, il sert à mettre en avant le barrage voûte dans son environnement montagneux. Le calque met en surbrillance non seulement le barrage dans sa structure bâtie, mais également la probable étendue qui constituait la retenue d'eau, aujourd'hui totalement comblée par plus de deux mètres d'alluvions. Dans un deuxième temps, un autre jeu interactif cherche à faire comprendre les différents éléments de construction visibles dans l'arrachement du barrage en partie gauche ${ }^{45}$. Le jeu de calques interactifs permet alors, au passage de la souris sur

\footnotetext{
lbid., p. 27
}

t5. Lin effet, un petit ruisseau, ténu mais persistant et provenant de différentes résurgences situées en amont, coule inexorablement en partie gauche du barrage, et a entraîné l'arrachement d'une partie de l'ouvtage. Si bien que celui-ci présente sur ce côté une sorte de coupe naturelle permettant de distinguer parfaitement bien un grand nombre des éléments constructifs du barrage voûte. 
chacun des éléments, de les mettre en avant. Le choix a été fait ici de proposer des calques en surimpression présentant des croquis des éléments à illustrer : la canalisation, la dalle de fond du canal, la maçonnerie, le parement autobloquant. Un troisième jeu de calques interactifs permet de mettre en évidence les premières hypothèses formulées par l'équipe de recherche. L'ouvrage est en effet constitué de deux parties distinctes : les vestiges d'un mur rectiligne en partic droitc, sur lesquels vient s'appuyer la partie droite du barrage voûte. L'hypothèse est ainsi émise de deux états de construction : un premier barrage droit, arraché en grande partie, puis la construction d'un barrage voûte, probablement beaucoup plus solide. Ainsi, au passage de la souris sur les textes soulignés en bleu indiquant les hypothèses, viennent en surimpression sur l'image les calques illustrant ces hypothèses. Enfin, puisque l'un des objectifs de la mission de recherche est d'établir précisément les tracés des aqueducs, un dernier jeu de calques interactifs permet de rendre compte de l'avancement des recherches : une première hypothèse de tracé vient en surimpression sur la carte.

En termes d'évaluation, indiquons que cet hypermédia a recueilli un accueil très favorable, non seulement de la part des différents membres de l'équipe avec qui il a été conçu, mais aussi de la part de nombre de personnes ayant assisté à la conférence donnée à Siliana, qui sont venues dire à tel ou tel d'entre nous combien l'élément visuel interactif, certes manipulé par un membre de la mission, était un soutien et complément important pour la compréhension du discours scientifique délivré en conférence.

3.5. Vers la professionnalisation du médiateur hypermédia du patrimoine archéologique

Ici, nous voulons insister sur la place accordée à la mise en valeur hypermédia au sein de cette mission, qui traduit une mutation d'ordre socio-professionnel sous l'influence des techniques digitales : il est relativement rare qu'une équipe archéologique scientifique inclut dès les étapes de travail sur le terrain des préoccupations claires de mise en valeur hypermédia du patrimoine, encore plus rare qu'elle fasse appel à un spécialiste de la médiation archéologique hypermédia dans les campagnes de fouilles. Ceci est intimement corrélé aux accords cadres passés entre les autorités tunisiennes et l'équipe de fouille, accords qui 
prévoient spécifiquement un important effort de la partie française concernant ces questions. Le contexte archéologique de cette mission de fouille est particulièrement riche, presque exceptionnel. Cet environnement favorable fournit de solides bases pour envisager la réalisation future de dispositifs hypermédias de valorisation archéologique touristique. L'association dès les étapes de fouilles d'un spécialiste de la médiation hypermédia du patrimoine est révélatrice de véritables (r)évolutions dans la prise en compte de ce secteur d'activité, sous l'impulsion à la fois de besoins touristiques et de la prise de conscience des potentialités des hypermédias en la matière. Il apparait probable que de plus en plus de missions de fouille concernant des sites archéologiques importants s'inscrivant potentiellement dans des circuits touristiques feront appel à des spécialistes de la médiation hypermédia du patrimoine archéologique. C'est à ce titre que les connaissances pratiques, de terrain, acquises par la participation à la fouille et aux prospections se révèlent tout à fait importantes pour ancrer l'action du médiateur dans une rigueur scientifique qui pose les bases d'une saine collaboration avec les équipes scientifiques. En effet, la participation active à la fouille et au travail archéologique de terrain représente un atout majeur pour le médiateur hypermédia : elle permet de construire une réflexion (et donc une action) au plus près des préoccupations des archéologues. Mais elle permet aussi au médiateur de sentir "physiquement », par lui-même, la réalité du travail archéologique de terrain qui constitue, il faut le rappeler, l'origine scientifique des informations qui sont à mettre en valeur. L'acquisition d'une compétence de terrain, certes généraliste, mais bien réelle, paraît ainsi être presque obligatoire pour faire du médiateur un acteur connaissant la réalité de ce qu'il traite dans une optique de médiation. Elle ancre dans le «regard » des autres membres de l'équipe, et plus généralement des scientifiques avec qui le médiateur peut être amené à travailler, l'idée selon laquelle ce dernier n'est pas déconnecté de ce qui est le propre de leur profession, à savoir la recherche archéologique, mais bien au contraire en prise directe avec le terrain de fouille. Cette idée est exactement celle qui justifie l'intégration de l'auteur de ces lignes à une autre équipe de recherche sur le terrain, celle de la mission de fouille du site de Sovjan. 


\section{L'hypermédia comme "plate-forme " Internet de circulation des savoirs : le site Web du site archéologique de Sovjan en Albanie}

Le cadre général de cette expérience est celui d'une équipe archéologique désireuse de disposer d'un outil permettant à la fois de rendre compte rapidement des résultats de fouille auprès de leurs collègues, à l'issue de chaque campagne de terrain, et de diffuser de l'information vers un large public amateur. À la suite d'une rencontre avec Gilles Touchais ${ }^{46}$, qui dirige la mission, les hypermédias apparaissant à même de répondre à ces préoccupations, a été monté un projet d'intégration à l'équipe, sous forme d'une commande d'un site Web et à partir d'une présence sur place de deux semaines à chaque campagne. La mission de Sovjan (École Française d'Athènes et Institut Archéologique de Tirana) travaille sur des habitats lacustres préhistoriques et protohistoriques, avec la particularité suivante : le site est en terrain humide et en partie submergé, et une fois la fouille terminée, les sondages sont très majoritairement rebouchés de sorte que rien n'est visible, alors même que les vestiges de constructions en bois datant d'environ 2200 av. J.-C. sont tout à fait importants et spectaculaires.

\subsection{Dispositif}

Le site Web est donc conçu ici comme une "plate-forme " de circulation des savoirs, entre savoirs scientifiques «bruts » et savoirs diffusés à destination d'un large public amateur. Pour répondre à cette orientation, trois modes de navigation sont proposés, correspondant à trois niveaux de lecture : un mode " découvrir ", un mode « approfondir » et un mode «professionnel ». Chacun des trois modes est accessible partout dans le site par un outil «mode » en partie supérieure gauche. Le premier mode permet de saisir les éléments essentiels du travail de la mission, en allant au plus direct et au plus facilement appréhendable par le grand public : localiser l'Albanie,

40 Professeur de protohistoire égéenne à l'Université de Paris l, LMR 7041 ArchScAn (Archéologie et Sciences de l'Antiquité), Maison de l'archéologie et de l'eth nologie de Nanterre. 
localiser le site, voir les vestiges principaux des maisons en bois, admirer quelques-uns des plus beaux objets issus des fouilles, comprendre en quelques mots l'importance des études paléo-environnementales qui font l'originalité de la mission. Le mode « approfondir » permet alors aux amateurs éclairés de rentrer très en détail dans le travail scientifique de la mission. Il ne s'agit plus seulement de localiser l'Albanie et le site, mais de comprendre sa topographie, la stratigraphie et les phases chronologiques qu'elle révèle. Il ne s'agit plus seulement de voir les principaux vestiges des maisons en bois, mais de comprendre comment ils se lisent sur le terrain (par un jeu de calques liant l'explication à l'iconographie et faisant apparaittre simultanément des animations sur une photo et sur un relevé des vestiges), quelles sont les principales connaissances que l'on peut en tirer concernant les techniques de construction (murs en clayonnage, sol, paroi, organisation spatiale, etc.), quelles sont les évolutions au cours des siècles et des périodes (évolution de l'habitat depuis le Néolithique jusqu'à l'Âge du Fer Ancien, la principale période étant représentée par l'Âge du Bronze Moyen), etc. Ce mode est aussi l'occasion d'apporter des éléments de connaissances complémentaires, par exemple sur l'historique de la recherche archéologique en Albanie, sur un bilan critique de l'Âge du Bronze en Albanie, sur les problématiques scientifiques générales de la mission, ou encore sur le récapitulatif précis de toutes les campagnes depuis 1993. Dans ces deux modes est largement utilisé le principe des calques interactifs, faisant apparaître sur les images les éléments essentiels au service de la compréhension du fond. Quant au mode «professionnel », il permet de diffuser des informations à destination de la communauté scientifique archéologique s'y intéressant : rapports de fouille ${ }^{47}$, information sur la base de données de la mission, sur la mise en place d'un système d'information géographique ou sur la bibliographie. Enfin, lui aussi partout présent dans le site, permet d'accéder à des pages présentant des liens Internet, la composition de l'ensemble de l'équipe, ou encore une page d'aide à la navigation.

Jusqu'à ce que ceux-ci soient officiellement publiés, plusieurs années aptès, dans le Bulletin de correspondance bellenique. 
4.2. Vers la transversalité de l'outil hypermédia : science et médiation mêlées sur le même support

C'est ici l'expérience où sont le plus directement liés, dans un même dispositif, savoirs "savants » et savoirs valorisés. Il n'est pas besoin d'argumenter plus avant sur le fait que ce sont les potentialités de l'outil hypermédia, en l'occurrence un site Web, qui permettent cette mixité. L'évolutivité du site Web, et donc la réactivité des informations produites par les scientifiques sont à coup sûr des atouts supplémentaires du dispositif Internet. Là encore, l'équipe scientifique exprime clairement la qualité de l'outil de médiation comme moteur de la recherche scientifique : pour créer les contenus, le médiateur oblige l'équipe à une perpétuelle remise au clair des connaissances qu'elle produit et de ce qu'elle comprend à partir des vestiges qu'elle met au jour. La lecture approfondie des quelques quinze rapports de fouilles, des articles et des nombreux documents de conférences et de travail, en bref le dépouillement de la documentation scientifique archéologique, est un des éléments du travail de conception ${ }^{48}$. Car en effet notre méthodologie de conception ne prévoit pas de demander aux archéologues de rédiger pour nous les contenus de médiation, mais bien de le faire nous-mêtme à partir de la matière scientifique brute. Ce faisant, le médiateur est un des seuls personnages de l'équipe à relire tout ce qui a été produit et écrit depuis presque quinze ans autour de ce site archéologique ${ }^{49}$ et il est fort naturel, les connaissances progressant au fil des campagnes et des découvertes, qu'il y trouve des imprécisions ou des contradictions. Cherchant à résoudre celles-ci (ou parfois au contraire à les mettre en lumière comme «moment» naturel de la progression de la recherche archéologique), il entame avec l'équipe des discussions de fond qui permettent pour l'archéologue de se replonger, sous un angle différent, dans l'objet de son travail quotidien. Les membres de l'équipe s'accordent à dire que la médiation est ainsi utile

4H D'où découlera le deuxième élément qui consistera en la structuration des contenus et en l'organisation de leur accessibilité.

49) On retrouve ici la question de la professionnalisation du médiateur hypermédia en archéologie, qui inclut la nécessaire connaissance approfondie de la recherche archéologique; en effet, le dépouillement correct d'une documentation scientifique exige une "compétence métier " seule à même de valoriser le discours scientifique. 
à la recherche archéologique elle-même, en permettant des jeux interactifs modifiables à volonté et en obligeant les concepteurs (médiateurs et archéologues) à se poser des questions interprétatives.

\section{L'hypermédia comme dispositif de valorisation touristique, de gouvernance et d'aménagement territorial et culturel : le site archéologique de Saint-Lézer}

\subsection{Description du dispositif}

Au cœur de la Bigorre en France, les importants vestiges d'un rempart du Bas-Empire, dont certaines parties du couronnement et du chemin de ronde sont exceptionnellement conservées, jonchent les flancs d'une colline qui fut d'abord un oppidum aquitain, puis le site fortifié du castrum Bigorra protégeant la civitas Tarba et la ville antique de Tarbes (située à 15 $\mathrm{km}$ au sud et dépourvue de fortifications connues), puis, enfin, un site médiéval pourvu d'une motte castrale dominant un petit village fortifié et un monastère dédié aux saints Félix et Lézer (ce dernier ayant donné son nom au village actuel).

Le contexte est donc ici celui d'une prestation de service ayant pour origine une commande qui porte la marque de préoccupations touristiques. Celles-ci s'inscrivent dans le cadre de politiques volontaristes d'aménagement territorial accordant une large place de valorisation du patrimoine et aux technologies digitales : le dispositif hypermédia commandé complète ainsi un aménagement physique du site sur lequel est proposé un sentier archéologique permettant de découvrir les vestiges en disposant d'informations sur de grands panneaux explicatifs. Au sein d'une modeste Communauté de communes essentiellement rurales, le projet des élus est porteur à la fois d'une dimension de proximité, de réappropriation de la culture, du patrimoine et de l'histoire locale, et d'une dimension nationale/internationale, qui vise à accroître la visibilité et l'attrait du territoire. "Exister culturellement sur le réseau et faire partager les richesses de notre patrimoine pour revitaliser notre territoire ", tel est clairement l'objectif fixé à l'hypermédia commandé. On est bien là au 
cœur d'une politique publique ${ }^{511}$ relevant d'un processus de gouvernance territoriale. La valorisation hypermédia du patrimoine engage pour les institutions qui en sont en charge des dimensions et enjeux socioculturels, mais aussi directement économiques, touristiques et stratégiques $^{51}$.

Les contenus s'organisent en quatre parties. La première concerne les éléments généraux de géographie et d'histoire. Un premier jeu de calques interactifs permet ainsi d'illustrer l'implantation du site, à vocation défensive, sur un contrefort dominant une vallée riche et fertile dans laquelle circulent deux cours d'eau. Un deuxième jeu permet, à partir d'une photo aérienne, de faire comprendre l'évolution de l'occupation du site. Ces pages utilisent des principes ergonomiques permettant, par un jeu de calques emboités qui apparaissent et disparaissent au passage de la souris (utilisation des fonctions show/ hide layers), de démultiplier les informations dans l'espace de l'écran sans alourdir la lecture ni proposer de pages très longues présentant un ascenseur sur la droite. Ainsi, à partir d'un menu chronologique, le passage de la souris fait apparaittre et disparaitre des blocs de textes qui contiennent eux-mêmes des liens interactifs faisant apparaître des éléments graphiques sur l'illustration. La deuxième partie présente le contexte de la recherche archéologique (historiographie, objectifs scientifiques, etc.), tandis que la troisième se consacre au rempart à proprement parler. L'une des pages s'attache alors à faire comprendre les principes constructifs de la muraille : une illustration d'un relevé en coupe sert à la fois d'outil de navigation dans les contenus et d'outil de visualisation. En passant sa souris sur les différentes parties de la muraille (fondations, soubassement, élévation, couronnement), l'utilisateur fait apparaittre un calque spécifique contenant des liens qui font à leur tour apparaitre des éléments graphiques sur le relevé en coupe. La quatrième partie présente les autres éléments patrimoniaux

54 Bruno Ory-Lavollée, "La diffusion numérique du patrimoine, dimension de la politique culturelle. Rapport au Ministère de la culture et de la communication ", Paris, 2002.

51 Bernard Miège, "Ies TICs : un champ marqué par la complexité et un entrelacs d'enjeux ", L'information-communication, abjet de connaissance, Bruxelles, De Boeck Université, 2004, p. 113-123 (publication originale : conférence au colloque BOGUES 2001, Montréal, av ril 2002). 
présents dans le village de Saint-Lézer (le prieuré, le moulin, le four à chaux du XVII ${ }^{c}$ siècle, etc.). Enfin, partout présent dans le site, un outil " annexe " permet d'accéder à un glossaire, à un répertoire de liens vers d'autres sites Web ou encore aux informations touristiques pratiques.

Le site Web est rattaché au propre site de la Communauté de communes, ce qui permet de fournir un premier élément d'évaluation basé sur la mesure de fréquentation de ce site Web communal : en effet, disposant d'un système de statistiques de fréquentation, le site Web de la Communauté de communes a connu une augmentation de fréquentation de $41 \%$ sur le mois suivant la mise en ligne et les opérations de promotion du site Web de Saint-Lézer (présentation publique). Par ailleurs, sans pouvoir être mesuré précisément car ne disposant ni d'un guide-conférencier ni d'un système de perception de droits, les visites physiques du site archéologique ont considérablement augmenté ${ }^{-2}$, notamment en raison de plusieurs visites scolaires organisées par des écoles du département auxquelles avaient été envoyé un CD-Rom complétant le dispositif $\mathrm{Web}^{5.3}$.

\subsection{Complexité exogène des hypermédias : expression d'une perspective sociologique}

Nous proposons ici de porter un regard sur l'environnement complexe de réalisation de ce dispositif hypermédia.

On observe d'abord un phénomène de multiplicité : multiplicité des domaines impliqués dans le processus (archéologie et patrimoine, informatique, médiation); multiplicité des acteurs (producteurs [élus et collectivités territoriales], médiateur/concepteur, scientifiques, utilisateurs grands publics, touristes), multiplicité des processus en jeu (accès au savoir, gestion de l'information scientifique, démocratisation technologique et culturelle, gouvernance territoriale, etc.) Dans notre cas, c'est une Communauté de communes (institution publique administrative en charge de la gestion du patrimoine) qui compte sur son territoire un important site archéologique. Celui-ci est classé et protégé

52 Données obtenues par l'intermédiaire de l'Office du tourisme local et par les responsables de l'association "Bigorra ", très souvent présents sur le site.

5.3 Celui-ci reprend les contenus du site Web en approfondissant certaines questions. 
par le Service régional de l'archéologie (autre institution publique en charge, elle, de la protection), et il est étudié par des équipes d'archéologues et architectes (scientifiques qui relèvent du monde de la recherche, des universités et des laboratoires). Il est ouvert aux visites touristiques et scolaires et, à ce titre, il peut intéresser des toursopérateurs et agences touristiques, aussi bien que les éditeurs de guides et les enseignants. Les opérations de médiation culturelle hypermédia, elles, intéressent les spécialistes de la médiation culturelle aussi bien que les entreprises de multimédia et autres agences de développement Web. Ces derniers travailleront à partir de la matière scientifique fournie par les archéologues et architectes, dans la limite de ce qu'imposent les nécessités de protection du site, en fonction des besoins précis exprimés par la mairie qui joue ici le rôle de client ayant passé une commande, et selon leur connaissance propre des processus cognitifs mis en jeu par les hypermédias et leur propre capacité à dépouiller la documentation scientifique. Ce CD-Rom et ce site Internet, en mettant en valeur la connaissance historique relative au site archéologique, participe à un processus de valorisation scientifique aussi bien qu'à une action en faveur de la promotion touristique, et donc de l'aménagement du territoire. Nous sommes bien en présence d'une situation qui met en jeu de multiples acteurs et de multiples processus. Par ailleurs, le produit final de cette situation de médiation hypermédia du patrimoine archéologique est le fruit d'interrelations complexes entre la mairie, le Service régional de l'archéologie, les responsables scientifiques des fouilles et études, les experts en médiation culturelle hypermédia, mais aussi entre le besoin de promotion touristique, la transmission pédagogique et la réalité économique du coût de développement d'un CD-Rom ou d'un site Web. Sur un autre versant, ont peut décrire l'existence de ce phénomène particulier de communication dans un environnement, que constitue le méta-système anthropo-social dans lequel se déroulent les actions de médiation hypermédia du patrimoine. Dans notre exemple, imaginons une association des amis du patrimoine archéologique de la commune qui organise une journée découverte, un enseignant de Latin au collège de la commune qui souhaite faire travailler ses élèves sur l'Antiquité au travers des vestiges conservés sur le site et, enfin, une famille qui voyage dans la région et souhaite se divertir tout en visitant un lieu historique et en faisant une belle ballade. Ces trois groupes se retrouvent sur le site archéologique. Chacun le 
visite en fonction de ses objectifs propres, mais tous sont susceptibles de consulter le site Web ou d'acquérir le CD-Rom archéologique. Lors de l'utilisation de l'hypermédia, l'appréhension réelle de chacun sera fonction de ses codes culturels, de ses connaissances historiques, de ses capacités cognitives, de son aisance dans l'utilisation des techniques digitales, etc. Ce sont ces caractéristiques qui constituent le contexte technico-socio-culturel dans lequel s'exprime l'action de médiation hypermédia du patrimoine archéologique.

\section{Conclusion}

Les voies qui ont été esquissées ici portent potentiellement, nous semble-t-il, quelques perspectives nouvelles de recherche. Les champs des sciences historiques et archéologiques, des sciences de l'information et de la communication, de la sémiologie, des sciences de la cognition, des sciences de l'éducation, des sciences de la conception ont été conjointement convoqués, parce que tous partie prenante d'un phénomène qu'aucun n'est à même de prendre en charge seul. L'on veut croire que chacune de ces disciplines peut transcender les cadres traditionnellement disjoints pour participer à la construction d'un projet de recherche et à l'enrichissement des outils de pensée que celui-ci requiert. La valorisation scientifique hypermédia perçue comme un processus technico-socio-cognitif complexe nous permet ainsi de renouveler nos manières traditionnelles d'envisager l'activité de médiation des connaissances scientifiques (ici archéologiques). Pour peu que chaque acteur impliqué veuille bien y reconnaitre un champ d'investigation nouveau, qui se construit peu à peu, les hypermédias peuvent être amenés à jouer un rôle de plus en plus important dans les activités de médiation et valorisation scientifique, qui font, au final, une bonne part de la noblesse de l'activité de recherche scientifique. Récursivement, l'on veut croire également que ce projet peut participer à la construction permanente des épistémologies qui renouvellent notre entendement collectif des phénomènes perçus complexes. 


\section{Bibliographie}

Akrich, Madeleine, "Les formes de la médiation technique ", Réseaux, n 60, 1993 , p. $87-98$.

Bachelard, Gaston, La formation de l'esprit scientifique, Paris, Vrin, 1938.

Bachelard, Gaston, Le nouvel esprit scientifique, Paris, PUF, 1934.

Breton, Philippe, Le culte de l'Internet, Paris, La Découverte, 2000.

Castells, Manuel, La galaxie internet, Paris, Fayard, 2001.

Castells, Manuel, La société en réseaux, Paris, Fayard, 1998.

Chambat, Pierre, "Usage des TIC : évolution des problématiques ", Technologies de l'information et société, vol. $6, \mathrm{n}^{\circ} 3,1994$, p. 249-270.

Davallon, Jean, Hana Gottesdiener et Joëlle Le Marec, "Approche de la construction des usages de cédéroms culturels liés aux musées ", Université Jean Monnet, Centre d'étude et de recherche sur les expositions et les musées, Ministère de la culture, Direction des musées de France / Réunion des musées nationaux, Paris, 1997.

Jacobi, Daniel, Diffusion et vulgarisation : itinéraire du texte scientifique, Annales littéraires de l'Université de Besançon, Les Belles Lettres, 1986.

Jeanneret, Yves, Écrire la science - Forme et sens de la vulgarisation, Paris, PUF, 1994.

Jeanneret, Yves, Joëlle Le Marec et Emmanuel Souchier, Lire, écrire, récrire. Objets, signes et pratiques des médias informatisés, Paris, BPI / Centre Pompidou, coll. "Études et recherche ", 2003.

Jouët, Josiane, « Retour critique sur la sociologie des usages », Réseaux, n 100, 2000, p. $487-521$.

Julia, Jean-Thierry et Emmanuelle Lambert, "Énonciation et interactivité : du réactif au créatif », Communication et langages, $\mathrm{n}^{\circ} 137,2003$, p. 30-44.

Kuhn, Thomas, La structure des révolutions scientifiques, Paris, Flammarion, 1972.

Lacroix, Jean-Guy, et al., "Industries culturelles et informatisation sociale - Nouvelles perspectives de recherche ", Sciences de la société, $\mathrm{n}^{\circ} 40$, Toulouse, PUM, 1997, p. 39.

Le Bæuf, Claude et Nicolas Pelissier, "Communiquer dans la complexité », dans Claude Le Bceuf et Nicolas Pelissier (dir.), Communiquer l'information scientifique Éthique du journalisme et stratégies des organisations, Paris, L'Harmattan - Série communication et technologie, 2003, p. 13-18.

Le Moigne, Jean-Louis, Le constructivisme, tomes 1 à 3, Paris, L'Harmattan, coll. "Ingénium ", 2001, 2002, 2003.

Mahoudeau, Julien, Médiation des savoirs et complexité. Le cas des bypermédias archéologiques et culturels, Paris, L'Harmattan, coll. «Ingénium ", 2007.

Mallein, Philippe $t$ al., «Multimédia et significations d'usage : les critères sociologiques de qualité d'usage du multimédia, le cas d'Internet ", dans Michel Dubois (dir.), Étude sociocognitive des usages du multimédia, ERIHST-CERAT / CNRS / CNET, janvier 1998.

Miège, Bernard, "Les TICs : un champ marqué par la complexité et un entrelacs d'enjeux ", dans L'information-communication, objet de connaissance, Bruxelles, De Boeck Université, 2004, p. 113-123 (publication originale : conférence au colloque BOGUES 2001, Montréal, avril 2002). 
Ory-Lavollée, Bruno, "La diffusion numérique du patrimoine, dimension de la politique culturelle. Rapport au Ministère de la culture et de la communication », Paris, 2002.

Proulx, Serge, "Usages des technologies d'information et de communication : reconsidérer le champ d'étude? ", dans Émergence et continuité dans les recberches en information et communication - XI Congrès international des sciences de l'information et de la communication, janvier 2001, UNESCO (Paris), SFCIC, 2001, p.64-65, http://grm.uqam.ca/textes/proulx_SFSIC2001.pdf, site consulté en mars 2007.

Proulx, Serge et Michel Sénécal, "L'interactivité technique, simulacre d'interaction sociale et de démocratie? "Tecbnologies de l'information et société, vol. $7, \mathrm{n}^{\circ} 2,1995$, p. $239-255$

Roqueplo, Philippe, Le partage des savoirs. Science, culture, vulgarisation, Paris, Seuil, 1974.

Séguy, Françoise, Les produits interactifs et multimédias - Métbodologies - Conception - Écritures, Grenoble, Presses universitaires de Grenoble, coll. « La Communication en Plus », 1999.

UTAH, "Recherches sur l'alimentation en eau à Zama dans l'Antiquité. Bilan et perspectives après la campagne de septembre 2003 ", Rapport de la mission tunisofrançaise de fouille à Zama, Unité toulousaine d'archéologie et d'histoire, UMR 5608 CNRS, Toulouse, 2003, p.1 à 26.

Vidal, Geneviève, "L'interactivité et les sciences de l'information et de la communication ", Actes du XVII' congrès des sociologues de langue française - Tours, juillet 2004, Groupe de travail Sociologic de la communication, 2004, p. 366-375.

\section{Références Internet}

http://www.vic-montaner.com/patrimoine/st-lezer/index.htm, site consulté en juillet 2007.

http://www.sovjan-archeologie.net, site consulté en juillet 2007. 